\title{
Prediction of Surface Roughness Using Artificial Neural Network in Single Point Diamond Turning
}

Engineering

KEYWORDS : ANN, Surface roughness,

Single Point Diamond Turning, Back propagation.

\section{Anandhan M \\ Department of Manufacturing Engineering, College of engineering Guindy, Anna University, Chennai, Tamilnadu, India \\ Rama Gopal V Sarepaka \\ Hariharan P \\ Central Scientific Instrumentation Organization-Central Scientific and Industrial Research, Chandigarh, India \\ Department of Manufacturing Engineering, College of engineering Guindy, Anna University, Chennai, Tamilnadu, India}

\section{ABSTRACT}

In manufacturing industries, manufacturers focused on the quality and productivity of the product. To increase the productivity of the product, computer numerically machine tools have been implemented during the past decades. Surface roughness is one of the most important parameters to determine the quality of product. The mechanism behind the formation of surface roughness is very dynamic, complicated, and process dependent. Several factors will influence the final surface roughness in a Diamond turning operations such as controllable factors (spindle speed, feed rate and depth of cut) and uncontrollable factors (tool geometry and material properties of both tool and work piece). Some of the machine operator using trial and error method to set-up machine cutting conditions. This method is not much effective and efficient and the achievement of a desirable value is a repetitive and empirical process that can be very time consuming. In order to solve the problem, a surface prediction technique based on artificial neural network prediction models is developed to predict the machining response for different input machining parameters. Thus, manufacturers can improve the quality and productivity of the product with minimum cost and time.

\section{INTRODUCTION}

In modern day world precision is a necessary need. The surface finish enhances the performance and economical aspects of a product. As the technology move towards next decimal place the ultra high precision of a product is must, without which the technology does not advances further. Though the most of the manufacturing process are very complex processes which involves cutting, ploughing, burnishing and material swelling, nanometric surface roughness is the output needed from ultra precision manufacturing processes [1]. The developed theories for bulk machining failed at nano level. Among few ultra precision machining processes, Single Point Diamond Turning (SPDT) produces better nanometric surface with lesser surface defects. SPDT is widely used in machining of all precision components. The single point contact of diamond tool induces less tool marks and residual stress than other machining processes. Almost all machining parameters can be controlled in modern day manufacturing, but few parameters like material properties, environmental factors cannot be controlled all the way. With the uncertainty in nano level surface finish due to uncontrollable factors, the prediction of surface with the machining parameters is a tidy task. The prediction before actual machining provides manufacturers with a realistic estimate of machining involved and support to their time and work would not be in vain.

The real time mathematical modeling of surface roughness needs much effort and skills to accomplish, which is the reason behind the development of prediction models using artificial neural network. A sound prediction of surface roughness is the goal of many researchers. Recent works showed that several neural network models to perform predictions. To improve and optimize the turning process, controllers were developed analytically and empirically [2]. Nevertheless, nonlinear relations among parameters restrict validation. In SPDT, cutting speed, feed rate and depth of cut are the important parameters which influence the surface roughness. The dynamic motion between work and tool also depends on these parameters. The uncertainty brings several economical and environmental disadvantages. The surface roughness defines the geometry of the surface. There are number of roughness parameters that are specified to designate the surface. The surface can be characterized by two main parameters, average roughness $(\mathrm{Ra})$ and peak to valley height waviness $(\mathrm{Pt})$.

This paper presents a strategy for Ra and Pt prediction based on the measurement of surface roughness with different pa- rameters consists of depth of cut, cutting speed, tool feed rate. To determine the network structure, performance of network consisting of constant number of input (3) and output (2) layer neuron is measured with respect to changing number of hidden layer neurons between 1-20. The network with 3-9-2 neurons per layer which has Mean Square Error (MSE) of 0.054303 at the $8^{\text {th }}$ epoch is chosen. The network is validated with test data and some suggestions for improvement and future works are given.

\section{EXPERIMENTATION SETUP}

Data were obtained from experiments conducted on a Single Point Diamond Turning machine (Nanoform250 - Taylor Hobson made) at the National Aspheric Facility of Central Scientific Instruments Organization (CSIR-CSIO), Chandigarh, India. The schematic of SPDT is shown in Fig. 1.

The work piece material used was selected to represent the majority of materials used in industries. For this study Aluminum 6061 was used. The specimen was a cylindrical bar with $50 \mathrm{~mm}$ diameter and $30 \mathrm{~mm}$ length. Before the experiments, the specimens were turned in CNC lathe to the aspheric shape with $1 \mathrm{~mm}$ depth of cut to remove outer layer. The surface discontinuities are removed by prior machining. The cutting diamond tool by Contour fine tooling company of nose radius $0.508 \mathrm{~mm}$ and shank height of $10.677 \mathrm{~mm}$ were used. Cutting parameters, i.e. cutting speed, feed rate and depth of cut were chosen such a way that the machining behavior can be easily studied. Each experiment was carried out with new sharp tools in order to keep the cutting conditions unchanged. The details of machining are given in Table 1.

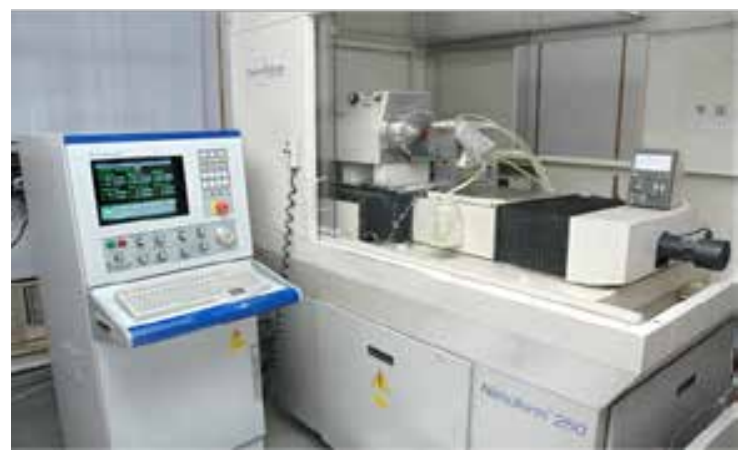

Figure 1: Single Point Diamond Turning machine 
TABLE - 1

\section{LEVELS OF EXPERIMENT}

\begin{tabular}{|l|c|c|c|c|}
\hline Consrollable Factor & Chit & Level I & Level 2 & Lewel 3 \\
\hline Depth of Cat & $\mu \mathrm{m}$ & 5 & 10 & 15 \\
\hline Spindle Speed & $\mathrm{Rpm}$ & 2000 & 3000 & 5000 \\
\hline Feed Rate & $\mu \mathrm{m} / \mathrm{rev}$ & 3 & 6 & 10 \\
\hline
\end{tabular}

Tool feed rate of $15 \mu \mathrm{m} /$ rev was added for testing the network. Therefore total experiments were performed are 36. For the needs of training and testing the created ANN the whole experimental data set $\left(\mathrm{N}_{\mathrm{tot}}=36\right)$ is randomly divided into a data subset for training $\left(\mathrm{N}_{1}=27\right)$ and a data subset for testing the ANN $\left(\mathrm{N}_{2}=\right.$ 9). The Contact profilometer (PGI 120 - Taylor Hobson made), a surface roughness measuring instrument is employed for measuring average surface roughness ( $\mathrm{Ra}$ ) and peak to valley height waviness (Pt). The PGI 120 is equipped with the diamond tip of $2 \mu \mathrm{m}$ in diameter. An average of three scans at an angle of $120^{\circ}$ to each other was used as a response value [3]

\section{ARTIFICIAL NEURAL NETWORK (ANN)}

ANNs has building blocks called neurons which connect input to the output. Computing with neural network is non-algorithmic. They are trained through examples rather than programs. Well explained information can be found in [2]. This paper used ANN approach to develop an efficient prediction model for surface roughness based on given input cutting conditions.

TABLE 2

DIAMOND TURNING RESULTS FOR TRAINING ARTIFICIAL NEURAL NETWORK

\begin{tabular}{|c|c|c|c|c|c|}
\hline Combi $N \theta$ & $\begin{array}{c}\text { Criming Speed } \\
\text { (opuy }\end{array}$ & 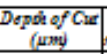 & \begin{tabular}{|c|} 
Tool Food \\
gess (umprov)
\end{tabular} & 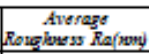 & 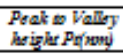 \\
\hline 1 & 2000 & \begin{tabular}{|l|}
5 \\
\end{tabular} & 3 & 29 & 337 \\
\hline 2 & 2000 & 5 & 6 & 30 & 486 \\
\hline 3 & 2000 & 5 & 10 & 30 & 370 \\
\hline 4 & 2000 & 10 & 3 & 31 & 402 \\
\hline 5 & 2000 & 10 & 6 & 28 & 442 \\
\hline 6 & 2000 & 10 & 10 & 31 & 497 \\
\hline 7 & 2000 & 15 & 3 & 33 & 577 \\
\hline 8 & 2000 & 15 & 6 & 29 & 425 \\
\hline 9 & 2000 & 15 & 10 & 32 & 481 \\
\hline 10 & 3000 & 5 & 3 & 31 & 381 \\
\hline 11 & 3000 & 5 & 6 & 32 & 332 \\
\hline 12 & 3000 & 5 & 10 & 31 & 395 \\
\hline 13 & 3000 & 10 & 3 & 30 & 443 \\
\hline 14 & 3000 & 10 & 6 & 28 & 483 \\
\hline 15 & 3000 & 10 & 10 & 32 & 452 \\
\hline 16 & 3000 & 15 & 3 & 28 & 459 \\
\hline 17 & 3000 & 15 & 6 & 32 & 534 \\
\hline 18 & 3000 & 15 & 10 & 30 & 388 \\
\hline 19 & 4000 & 5 & 3 & 31 & 321 \\
\hline 20 & 4000 & 5 & 6 & 31 & 332 \\
\hline 21 & 4000 & 5 & 10 & 32 & 423 \\
\hline 22 & 4000 & 10 & 3 & 29 & 508 \\
\hline 23 & 4000 & 10 & 6 & 31 & 501 \\
\hline 24 & 4000 & 10 & 10 & 25 & 489 \\
\hline 25 & 4000 & 15 & 3 & 24 & 412 \\
\hline 26 & 4000 & 15 & 6 & 25 & 470 \\
\hline 27 & 4000 & 15 & 10 & 23 & 629 \\
\hline
\end{tabular}

If a neural network is going to be effective, the training dataset must be complete enough to satisfy the following goals [4]:

Every group must be represented: The training dataset consists of several subgroups, each having its own central tendency toward a particular pattern. All of these patterns must be represented.
Within each class, statistical variation must be adequately represented: The range of data presented to the neural network must represent the entire range of data with noise included.

In certain cases, only lower and upper bound data with few scattered data points in between the range will be available. In that case, it is acceptable to have a sparse dataset.

Supervised networks are used for successful prediction. A back propagation (BP) algorithm was used with the experimental data. The BP network is a continuous valued, multi input-output feed forward layer that follows a The Levenberg-Marquardt method. The Levenberg-Marquardt method is a standard technique used to solve nonlinear least squares problems. Least squares problems arise when fitting a parameterized function to a set of measured data points by minimizing the sum of the squares of the errors between the data points and the function. The Levenberg-Marquardt curve-fitting method is actually a combination of two minimization methods: the gradient descent method and the Gauss-Newton method. The gradient descent method changes the weight proportional to the partial derivative of the error. The Gauss-Newton method, the sum of the squared errors is reduced by assuming the least squares function is locally quadratic, and finding the minimum of the quadratic. The back propagation phase changes the weight so that error of network can be minimized. This can be achieved by training input-target vectors into the network which manipulates output. The output is compared with target supplied and error value is calculated. The error adjusts the weights throughout the network. Thus if the same input-target are trained to the network, the error would be smaller than earlier found [5].

Neural network is used to learn the appropriate calibrating between input and output variables of the process. Average roughness ( $\mathrm{Ra}$ ) and peak to valley height waviness (Pt) are accepted as computable outputs with respect to inputs cutting speed, feed rate and depth of cut.

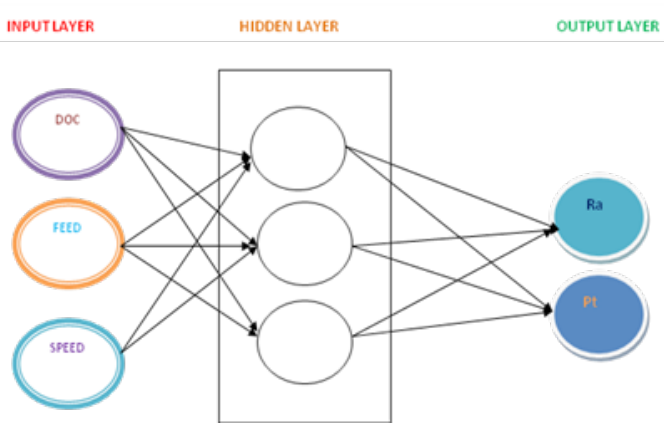

Figure 2: Architecture of neural network

Data containing 27 lines and 5 columns taken from the experiments is the supply data for neural network shown in Table 2 . Transfer functions are selected as tangent sigmoid in hidden layer and logarithmic sigmoid in output layer. Moreover Levenberg- Marqurdt method is chosen as training method. Then weights and bias values are updated according to this method. ANN model for predicting surface roughness is shown in Fig. 2.

\section{RESULTS}

The ANN's performance during training is measured according to the mean of squared errors (MSE). MSE is the average squared difference between outputs of the network and target values. Training is initially set to terminate after a maximum number of epochs (1000), but it is stopped at 17 iterations since no further improvement in the MSE is achieved. As depicted in Fig. 3., the prediction error, measured by the MSE, is low (i.e. 0.054303 ). 


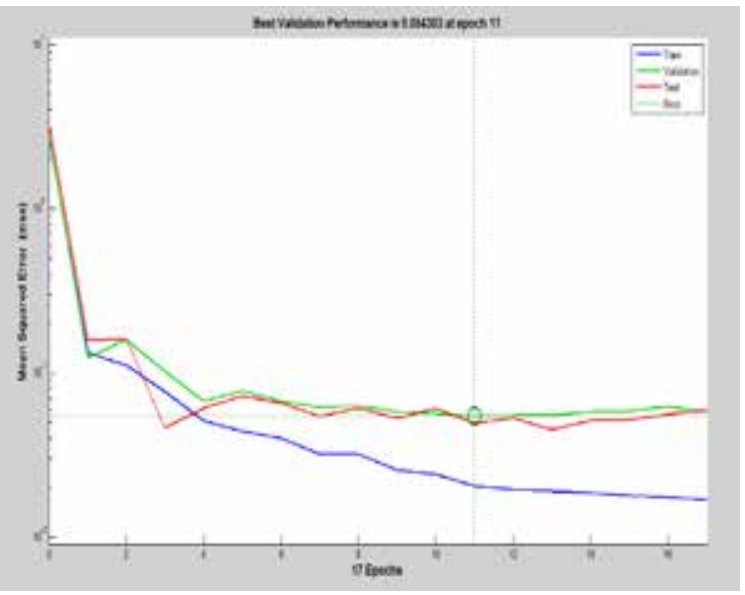

Figure 3: Mean square error $V s$. No. of epochs

Another performance measure for the network efficiency is the correlation coefficient (R). The correlation coefficient is a statistical measure of the strength of correlation between actual versus predicted values.

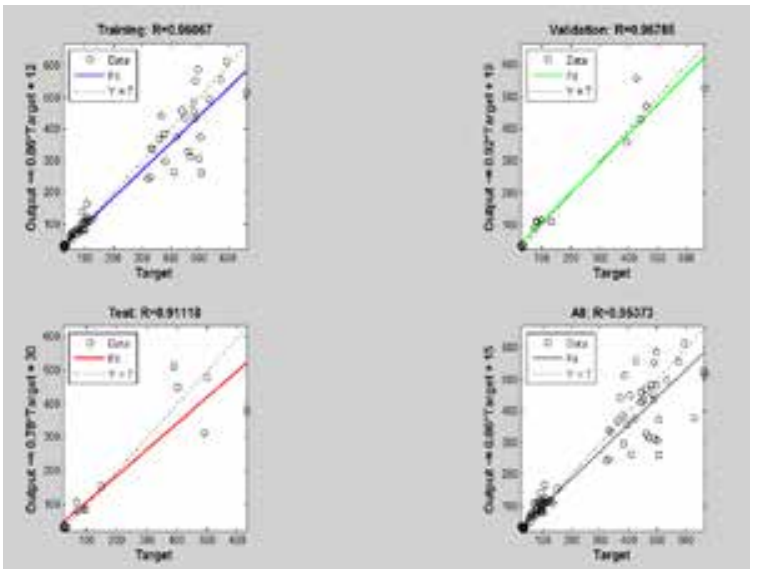

Figure 4: Regression coefficients (Target $V s$. Output)

As shown in Fig. 4., 3:9:2 neurons give the best values for Ra and $\mathrm{Pt}$ in the testing. In training regression coefficient $\mathrm{R}^{2}$ value becomes 0.96067 . It is shown that the predicted values are close to the experimental values. Apart from the L27 array, separate unknown data are tested with the network. The test data are tested with the network for prediction and the variation of data with respect to actual data are compared. The error in predicted value is given in the Tables 3 and 4 .

TABLE 3

PREDICTION ERROR FOR SURFACE ROUGHNESS

\begin{tabular}{|c|c|c|c|c|c|}
\hline Speed (rpwi) & $\begin{array}{c}\text { Drpul of cur } \\
\text { (pw iner) }\end{array}$ & $\begin{array}{c}\text { Tool } \\
\text { feed rate } \\
\text { (umin) }\end{array}$ & $\begin{array}{l}\text { Acrual } \\
\text { Ra(mai) }\end{array}$ & $\begin{array}{l}\text { Predicted } \\
\text { Rasimmo }\end{array}$ & S Error \\
\hline 2000 & 3 & 15 & 29 & 31.1394 & 7.3772 \\
\hline 2000 & 10 & 15 & 32 & 36.509 & 14.0906 \\
\hline 2000 & 15 & 15 & 36 & 33.7467 & -6.2592 \\
\hline 3000 & 3 & 15 & 34 & 30.5329 & -10.1974 \\
\hline 3000 & 10 & 15 & 35 & 29.5734 & -15.5046 \\
\hline 3000 & 15 & 15 & 35 & 33.2654 & -4.956 \\
\hline 4000 & 5 & 15 & 31 & 28.3898 & -8.42 \\
\hline 4000 & 10 & 15 & 38 & 36.4736 & -4.0168 \\
\hline 4000 & 15 & 15 & 31 & 30.8788 & -1.2903 \\
\hline
\end{tabular}

The change in predicted roughness with the actual roughness is shown in Fig. 5.

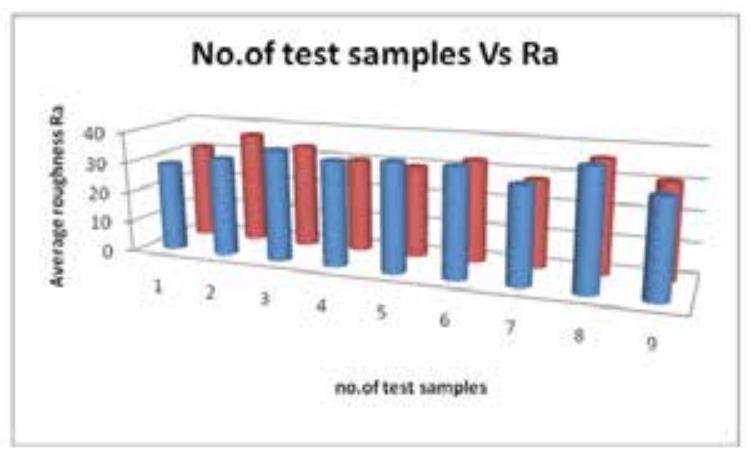

Figure 5: Fabricated surface roughness Vs. Predicted surface roughness

The change in predicted roughness for each combination of test data is shown in Fig. 6.

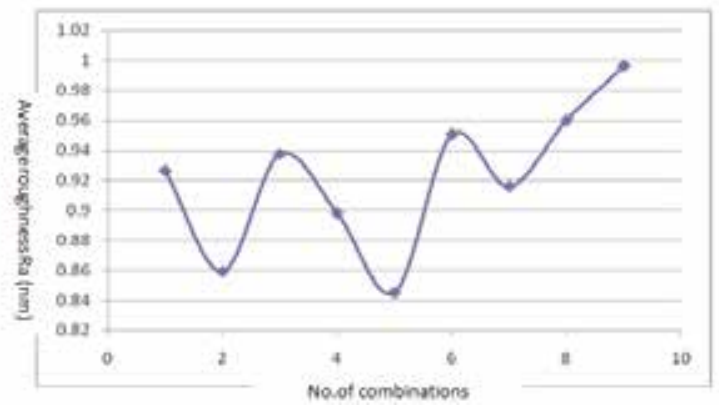

Figure 6: Change in Ra prediction error for different combinations

TABLE 4

PREDICTION ERROR FOR SURFACE WAVINESS

\begin{tabular}{|c|c|c|c|c|c|}
\hline Spered $(r p m)$ & $\begin{array}{c}\text { Deptit of owt } \\
\text { (pwawrey) }\end{array}$ & $\begin{array}{c}\text { Toof } \\
\text { freef rate } \\
\text { (rim) }\end{array}$ & $\begin{array}{l}\text { Actwal } \\
\text { PUTnem }\end{array}$ & $\begin{array}{l}\text { Profilitrd } \\
\text { Prines) }\end{array}$ & S Erer \\
\hline 2000 & 5 & 15 & 362 & 427.6058 & 18.1231 \\
\hline 2000 & 10 & 15 & 488 & 399.2665 & -18.1831 \\
\hline 2000 & 15 & 15 & 596 & 386.0752 & .35 .2223 \\
\hline 3000 & 5 & 15 & 463 & 568.8274 & 22.8569 \\
\hline 3000 & 10 & 15 & 666 & 308.8212 & -53.6305 \\
\hline 3000 & 15 & 15 & 661 & 403.5751 & -38.9448 \\
\hline 4000 & 5 & 15 & 380 & 401.1451 & 5.5645 \\
\hline 4000 & 10 & 15 & 500 & 690.2122 & 38.0424 \\
\hline 4000 & 15 & 15 & 504 & 411.2618 & -18.4004 \\
\hline
\end{tabular}

The change in predicted roughness with the actual roughness is shown in Fig. 7.

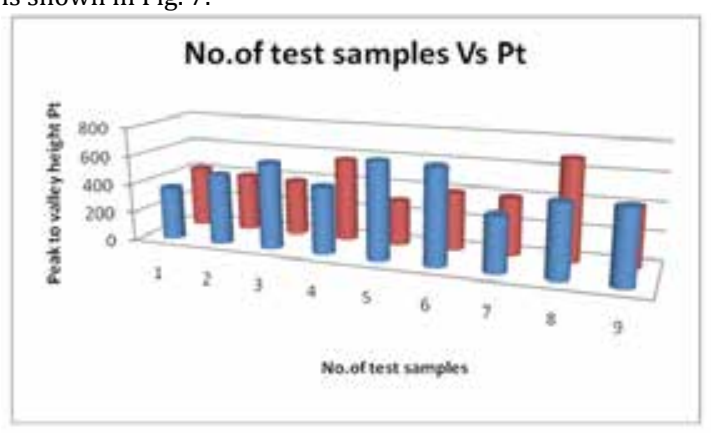

Figure 7: Fabricated surface waviness Vs Predicted surface 


\section{waviness}

The change in predicted peak to valley height waviness for each combination of tested data is shown in Fig. 8.

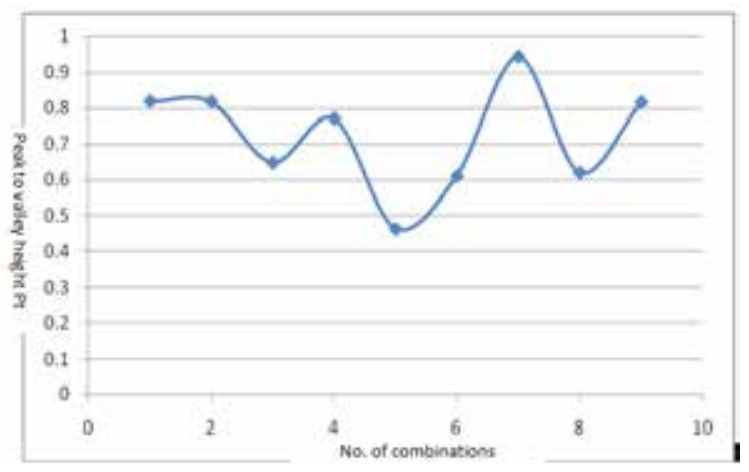

Figure 8: Change in Pt prediction error for different combinations

\section{CONCLUSION}

The experimental design and setup to develop an ANN prediction model for surface roughness have been developed. The model is devised for all cutting parameters using neural networks. Then the models are compared for their prediction capability with the actual values. The ANNs are used in decision making tool for complex manufacturing processes. Taking this paper as a foundation further research will be conducted to develop other prediction systems that could enhance the accuracy for surface roughness prediction.

\section{REFERENCE}

[1]. Cheung, C.F. and Lee, W.B., "Study of factors affecting the surface quality in ultra-precision diamond turning", Materials and manufacturing processes, Vol.15, No.4, pp.481-502 (2000). | [2]. Ezugwu EO, Arthur SJ, Hines EL.,"Tool-wear prediction using artificial neural networks". Journal of Material Processing Technology 49:255-264 (1995). | [3]. Neha Khatri, Vinod Mishra, Rama Gopal V Sarepaka., "Optimization of Process Parameters to Achieve Nano Level Surface Quality on Polycarbonate". International journal of computer applications. Vol.48 No.13,(2012). | [4]. Rajkumar T. and Jorge Bardina "Training data requirement for a neural network to predict aerodynamic coefficients" SAIC@ NASA Ames Research Center, Moffett Field, California, USA 94035., [ [5]. Manoj Kumar, Anirudh Biswas.," Application of Taguchi and A.N.N. method for optimization of machining parameters". International Journal of Mechanical, Civil, Automobile and Production Engineering, Vol. 3 No. 2, (2013). 